\title{
Temporal grouping and direction of serial recall
}

\author{
Yang S. Liu ${ }^{1}$. Jeremy B. Caplan²
}

Published online: 23 July 2020

(C) The Psychonomic Society, Inc. 2020

\begin{abstract}
When lists are presented with temporal pauses between groups of items, participants' response times reiterate those pauses. Accuracy is also increased, especially at particular serial positions. By comparing forward with backward serial recall, we tested whether the influence of temporal grouping is primarily a function of serial position or output position. Results favored the latter, both when recall direction was known to participants prior to (Experiment 1) or only after (Experiment 2) studying each list. Alongside fits of variants of a temporal distinctiveness-based model, our findings suggest that the influence of temporal grouping is not just a consequence of grouping information stored during the study phase. Rather, it critically depends on participants cueing with within-chunk position during recall, combined with response suppression.
\end{abstract}

Keywords Serial recall $\cdot$ Order memory $\cdot$ Memory models $\cdot$ Grouping $\cdot$ Response suppression

\section{Introduction}

Memory for sequences is widely understood to be at the core of a broad range of human behavior (e.g., Ebbinghaus, 1885/1913; Lashley, 1951). Accordingly, a major goal of memory research has been to determine how serial lists are stored and retrieved, with particular focus on the immediate serial-recall paradigm. In immediate serial recall, participants are presented with a list of items, such as consonants, for study, then asked to reproduce the list in order. This procedure resembles everyday tasks like remembering a phone number long enough to enter it into a smart phone or remembering a line of song lyrics long enough to repeat it in a pop concert. The effect of temporal grouping is prominent among the major empirical findings

Data can be accessed from https://osf.io/evmct/?view $\backslash$ protect_ only=be14d124209442f8882e476f812965b6

Electronic supplementary material The online version of this article (https://doi.org/10.3758/s13421-020-01049-x) contains supplementary material, which is available to authorized users.

Yang S. Liu

ly6@ualberta.ca

1 Department of Psychiatry, University of Alberta, Edmonton, AB T6G 2R3, Canada

2 Department of Psychology and Neuroscience and Mental Health Institute, University of Alberta, Edmonton, AB T6G 2E9, Canada that have been used to constrain and guide the development of mathematical models of serial recall. Introduced by Ryan (1969a, b), longer pauses are introduced between subgroups of items, while equating total list presentation time. For example, a list of nine consonants might be subdivided into three groups of three letters. Resulting plots of accuracy show a scalloping effect (e.g., Hitch et al., 1996; Ng \& Maybery, 2002; Ryan, 1969a; 1969b), with a relative recall advantage for the first and last item of each temporally defined group (e.g., items 1, 3, 4, 6, 7, and 9) compared the respective middle-group items.

The dominant theoretical account of the advantage of grouped over control lists is that grouping affords an additional cue participants can use during recall. This idea has been incorporated in numerous models that rely on positional or ordinal cueing for serial recall (e.g., Brown et al., 2000; Brown et al., 2007; Burgess \& Hitch, 1999; Henson, 1998). Thus, in the well-supported, ScaleIndependent Memory, Perception and LEarning model (SIMPLE; Brown et al., 2007), for example, control lists are recalled by cueing memory with time (specifically, studytest recency of the desired serial position), whereas grouped lists are recalled by cueing with a weighted sum of time and position of the desired item within the current group (e.g., 1,2 , or 3, for lists composed of groups of three items).

Backward serial recall can provide additional constraints on model mechanisms because it helps unravel effects of serial position versus response (output) position. However, we could find no published data on backward serial recall of temporally grouped lists. The closest precedent, 
to our knowledge, was by Anderson, Bothell, Lebiere, and Matessa (1998), who investigated both forward and backward recall of grouped and ungrouped lists. Latency measure showed long pauses across group boundaries, and the effect of grouping was similar between recall directions. Their lists were presented sequentially, but with groups denoted visually, both at study and test. In fact, serial position (of items within the whole list) were also cued spatially both during study and during recall. Thus, their outcome could have been due to explicit position cueing at study and test, similar to what was found by Guitard, Saint-Aubin, Poirier, Miller, and Tolan (2019). Here we present data from two experiments comparing temporally grouped and ungrouped lists, between forward and backward serial-recall directions. No indication of serial position was visually present during either study or recall. In Experiment 1, participants had foreknowledge of recall direction, and thus had the opportunity to customize their approach to study depending on direction. In Experiment 2, we prevented this by cueing serial-recall direction of each list only after the list had been studied.

We sought to test the standard account, where serial recall is cued by a combination of serial position (or time) and within-group position. Because the additional dimension of discriminability is a function of serial position, we predicted the benefit due to temporal grouping would be a function of serial position, and not of output position. The results of our first experiment ${ }^{1}$ directly contradicted this prediction. ${ }^{2}$ Participants either studied control lists or temporally grouped lists of nine consonants, and were either asked to recall the lists in order of presentation ("forward" recall direction) or in reverse order ("backward" recall direction). Both grouped/control and forward/backward direction were manipulated factorially between subjects. To anticipate the results, the benefits of temporal grouping were a function of output position (and the benefit did not appear at each position), rather than serial position (initial presentation order). That is, backward-recall serial position effects were nearly mirror images of the forwardrecall serial position effects. Experiment 2 was designed to test whether the effects would extend to participants who were post-cued for recall direction, to equate cognitive processes during study. The same basic outcome, the benefits of temporal grouping being a function of output

\footnotetext{
${ }^{1}$ Experiment 1 was, in fact, designed with an entirely different goal in mind: to provide comparison data for a study of judgements of relative order of temporally grouped lists, reported by Liu, 2015.

${ }^{2}$ Indeed, early attempts at fitting models to the aggregate data initially led us to conclude that this account was ruled out, the opposite of our ultimate conclusion here.
}

position rather than serial position, was once again found. The question then became, whether the standard account of temporal grouping could produce such a pattern. Our intuition was that it could not, because the benefit of the additional retrieval cue (within-group position) should be a function of serial position, regardless of order of recall. After presenting data from the two experiments, we report fits of variants of SIMPLE: first, using only time as a retrieval cue, to check whether the temporal pattern present during study, combined with participants' tendency to reiterate this pattern in their inter-response times during recall, might already be sufficient to explain the data. We then compare with the two-dimensional model, adding within-group position as a retrieval cue.

Finally, because backward serial recall is typically characterized by a recency-dominant serial position curve, rather than the primacy-dominant serial position curve found in the forward direction (e.g., Anderson et al., 1998; Bireta et al., 2010; Farrand \& Jones, 1996; Guérard et al., 2012; Guérard \& Saint-Aubin, 2012; Haberlandt et al., 2005; Li \& Lewandowsky, 1993; 1995; Madigan, 1971; St. Clair Thompson \& Allen, 2013; Thomas et al., 2003), we wondered if response suppression (participants' reluctance to repeat items in serial recall; Duncan \& Lewandowsky, 2005) might be an influential factor that could affect model fit.

In both experiments, temporal grouping was manipulated between subjects, as is typically done (e.g., Ryan, 1969a; 1969b; Hitch et al., 1996; Ng \& Maybery, 2002), because the presence of temporally grouped lists might induce participants to conceptualize ungrouped lists as grouped as well. Even so, some level of spontaneous grouping tends to be found even with ungrouped presentation procedures (e.g., Martin \& Noreen, 1974; Madigan, 1980; Jou, 2011; Wickelgren, 1967; Ryan, 1969a). The control condition was ungrouped only in the sense that presentation rate was not grouped, and the interpretation will rest upon the assumption that participants will organize lists into groups less in the control than grouped conditions. Recall direction was manipulated between subjects in Experiment 1. This had the advantage of allowing participants to optimize their performance on both backward and forward recall directions, but participants could have altered their study strategy between recall directions. To address this, recalldirection was manipulated within subjects in Experiment 2, by post-cueing direction. This ensured that participants could not study a list differently in anticipation of backward versus forward recall. Finally, fits of four variants of SIMPLE are presented, to directly test whether the standard account of temporal grouping might be able to accommodate our findings. 


\section{Experiment 1}

\section{Methods}

\section{Participants}

A total of 168 undergraduate students from introductory psychology courses at the University of Alberta participated in exchange for partial course credit. The sample size was selected to be close to a related experiment, testing judgements of relative order of the lists with the same design, and with the same between-subjects design with four groups (Liu, 2015, chapter 5). Participants gave informed consent, had normal or corrected-to-normal vision and learned English before age 6. Participants were run in groups of about 10 to 15 people, with random assignment to testing conditions as follows. Forward/Grouped: $N=41$; Forward/Control: $N=42$; Backward/Grouped: $N=43$; Backward/Control: $N=42$.

\section{Materials \& procedure}

To maintain continuity with previous studies (Chan, Ross, Earle, \& Caplan, 2009; Liu, Chan, \& Caplan, 2014), stimuli were 16 consonants (excluding $\mathrm{S}, \mathrm{W}, \mathrm{X}$, and Z) from the English alphabet displayed in uppercase. Each list comprised nine consonants drawn at random without replacement from the stimulus pool. Probability was equal for each consonant/serial-position combination. All participants were tested using a group of 15 computers (custom-built PCs) with identical hardware, identical Samsung SyncMaster B2440 monitors and Logitech K200 keyboards, to minimize hardware precision variability in our between-subjects design (Plant \& Turner, 2009).

The experiment was implemented with the Python Experiment-Programming Library (PyEPL; Geller et al., 2007). The procedure for the Control condition was identical to Liu et al.'s (2014) judgements of relative order experiment, but with list length 9 and a serial-recall test. Depending on grouping, participants were either asked to type the list in forward or backward order: (a) Excerpt from "forward" instruction: ". . . you will be asked to type the list you just saw, starting from the first letter and ending with the last letter. In other words, type the list in forward order. ..." (b) Excerpt from "backward" instruction: "... you will be asked to type the list you just saw, starting from the most recent letter and end with the first letter. In other words, type the list in backward order. ...". Participants were asked to press the SPACE key to skip over items they do not remember. Each trial began with a fixation asterisk, '*', in the center of the screen, followed by a consonant list presented sequentially in the center of the screen. Items were presented for $500 \mathrm{~ms}$ each. The Control condition had a constant inter-stimulus interval (ISI) of $350 \mathrm{~ms}$, whereas the Grouped participants had an ISI of $950 \mathrm{~ms}$ between items 3 and 4, and between items 6 and 7, to create a longer temporal gap between groups, and an ISI of $150 \mathrm{~ms}$ for all other transitions. The ISIs were selected to maintain a constant total presentation time of $7300 \mathrm{~ms}$ between Grouped and Control conditions (as done by, for example, Henson, 1998; Hitch et al., 1996). After a 2500-ms delay, participants were cued with an input line and a text reminder to type the list either in forward or backward direction. Participants could not backtrack to edit entered consonants, and they terminated recall by pressing the ENTER key. All letters of the English alphabet were accepted as input, and all typed letters stay on the computer screen until ENTER was pressed. After a 500-ms delay, participants could press a key to start the next trial. A total of 80 trials were presented in five blocks, where the first block contains eight practice trials, followed by four blocks of 16 trials.

\section{Data analysis}

We analyzed our data with linear mixed effects (LME) models (Baayen, Davidson, \& Bates, 2008; Bates, 2005). We adopted LME analysis because compared to ANOVA, LME can fit individual responses without the need for averaging of the data, and protects against Type II error due to increased power (Baayen et al., 2008; Baayen \& Milin, 2010). LME analyses were conducted in R (Bates, 2005), using the LME4 (Bates \& Sarkar, 2007), LanguageR (Baayen, 2007) and LMERConvenienceFunctions (Tremblay, 2013) libraries. The "Imer" function was used to fit the LME model. The "Anova" function from Companion to Applied Regression package (Fox \& Weisberg, 2011) was used to conduct Wald Chi-square test for the best-fitting models.

We conducted accuracy analyses with Serial Position (order of an item during presentation) included as a factor and then again with Output Position (response number within a given recall sequence) instead. Serial Position and Output Position were treated as a continuous fixedeffects predictors. Categorical fixed-effects predictors were Grouping and Recall Direction (between-subjects). Subject was included as a random effect on intercept. The accuracy data were fitted with logistic regression as it is a binary variable ("correct" vs. "incorrect"). Because the latency measure was an inter-response time, the time difference between two successive responses, we analyzed latency data only with respect to Output Position. Inter-response times leading into errors were excluded from the analysis. Interresponse time was log-transformed to reduce skewness.

LME estimates random effects first, followed by fixed effects. In the results tables, the "Estimate" column reports the corresponding regression coefficients, along with their 
standard errors. For the purposes of reporting the LME results, Control condition and Forward direction were set as the reference levels for their corresponding variables. The best fits of LME models were obtained by conducting a series of iterative tests comparing progressively simpler models with more complex models using the Bayesian Information Criterion (BIC), as was done by Liu et al. (2014), using LMERConvenienceFunctions developed by Tremblay (2013).

\section{Results}

Nine participants were excluded because they recalled fewer than two items on average (making their data uninformative with respect to serial-order, scalloping effects, etc.), as follows: Forward/Grouped: 0; Forward/Control: 2; Backward/Grouped: 1; Backward/Control: 6. First we comment on the global effects of the between-subjects factors on accuracy and response time, and then drill down into serial-position and output-position effects.

\section{Global effects of grouping and recall direction on accuracy} and inter-response time

We first plot the effects of Grouping and Direction, collapsing across Input/Output Positions (Fig. 1). For both accuracy and inter-response times of correct recalls, the basic pattern was the same: Backward recall was worse than forward recall, replicating numerous prior studies of backward recall. Also replicating prior temporal-grouping studies, Grouped lists produced higher accuracy (Fig. 1a) and faster inter-response times (Fig. 1b) than ungrouped, Control lists. The advantage of Grouped over Control was significant individually for Forward and Backward, both experiments, for accuracy. For inter-response time, the advantage of Grouped over Control was significant individually for Forward and Backward in Experiment 1, but for neither direction in Experiment 2. This pattern shows, for the first time, that grouping enhanced recall in the backward direction to a similar degree, overall, as in the forward direction. This may indicate that grouping
Fig. 1 Mean accuracy $(\mathbf{a}, \mathbf{c})$ and inter-response time $(\mathbf{b}, \mathbf{d})$ as functions of group for

Experiment $1(\mathbf{a}, \mathbf{b})$ and Experiment 2 (c, d). Error bars are $95 \%$ confidence intervals based on standard error of the mean. For both experiments, Grouped and Control were compared using Welch's $t$ test, as follows. Accuracy: Exp. 1 Forward:

$t(80.9)=2.74, p<0.01$,

Backward:

$t(82.9)=2.36, p<0.05$; Exp.

2 Forward:

$t(108)=4.49, p<0.01$,

Backward:

$t(108)=3.72, p<0.01$. IRT,

Exp. 1 Forward:

$t(64.4)=-2.59, p<0.05$,

Backward:

$t(82.7)=-2.18, p<0.05$;

Exp. 2 Forward:

$t(97.3)=-1.53, p=0.13$,

Backward:

$t(107)=-.03, p=0.98$ a

Accuracy

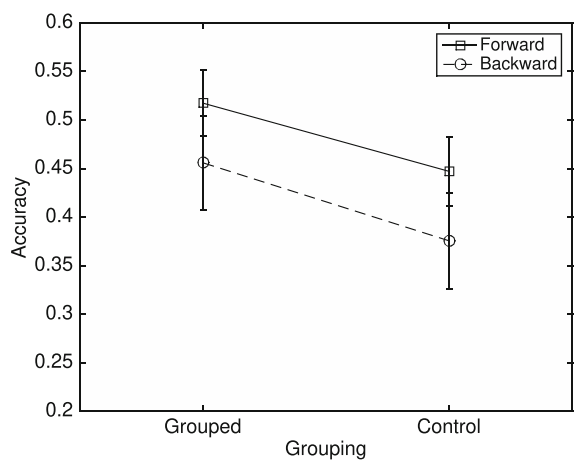

Experiment 1

b Inter-Response Time

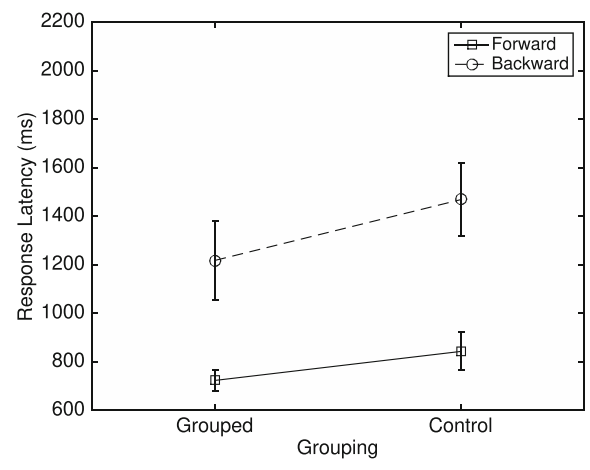

Experiment 2

c

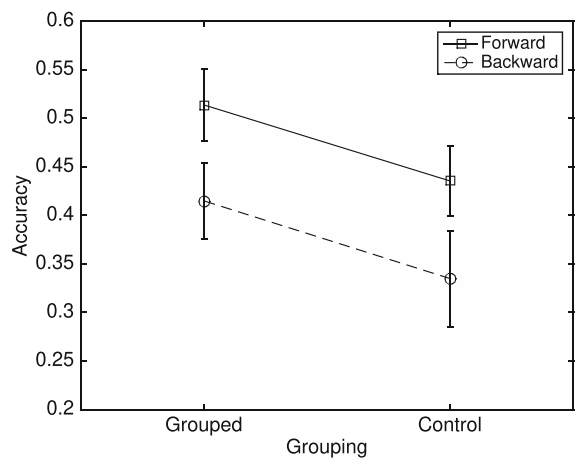

d

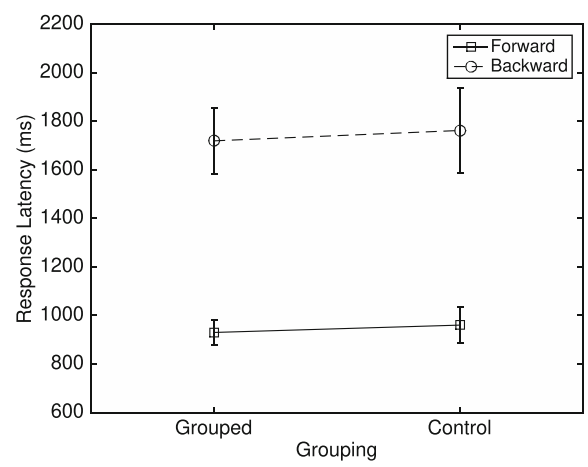


influenced backward recall via a similar mechanism as forward recall, but this could be due to effects at study or during recall. To address this, we next break down accuracy and inter-response time by serial position and then by output position.

\section{Do grouping effects depend on input or output position?}

Figure 2 plots serial-position curves for all four conditions. First, the grouped conditions show the characteristic scalloping pattern: mini U-shaped serial-position curves within groups (Ryan, 1969a). Unlike some previous results (e.g., Hitch et al., 1996), grouped lists do not exhibit an advantage at every serial position. This may be viewed as proof-of-principle that an account of temporal grouping that assumes position is encoded with greater resolution due to an additional, within-group position code, may be insufficient. At the very least, a weighting function may be required to explain such effects, as in SIMPLE, where the within-group weight and the temporal-dimension weight must sum to 1 (Brown, Neath, \& Chater, 2007). Next, the overall serial-position curves for backward recall are nearly (although not precisely) mirror images of the corresponding curves for forward recall, as previous studies have found. Moreover, the effects of grouping are approximate mirror images as well. Thus, the visual impression is that the benefit of grouping is not a function of serial position, but rather, of output position. The difference between the grouped and control conditions, plotted as a function of output position, confirms this, as it is quite similar for backward as for forward recall (Fig. 2c).

To check these effects, we fitted linear mixed effects models with either Serial Position (Table 1a) or Output Position (Table 1b), along with Grouping and Direction. Confirming visual inspection, results from the best-fitting linear mixed effects models found, on the one hand, a Grouping $\times$ Direction $\times$ Serial Position interaction, and on the other hand, no Grouping $\times$ Direction $\times$ Output Position interaction. To select between these two best models, the BIC values show that the best-fitting model using Output Position absent a three-way interaction fit better than the best-fitting model with Serial Position (which includes a three-way interaction): $\Delta \mathrm{BIC}=19$, comfortably surpassing the conventional criterion of $\Delta \mathrm{BIC}>2$.

Turning next to inter-response time as a function of output position (Fig. 3), all conditions showed the characteristic slow initiation time (Bireta et al., 2010; but see Thomas et al., 2003, for different results with backward recall). After the first recall, the Grouped participants had longer recall latencies across group boundaries, in exchange for shorter latencies within-group, compared to Control participants. This, too, held for both Forward and Backward recall directions. However, inter-response
Fig. 2 Recall accuracy as a function of serial position for forward recall (a) and backward recall (b). Accuracy difference of Grouped-Control as a function of output position for forward and backward recall (c). Error bars denote 95\% confidence intervals based on standard error of the mean. * $p<0.05$, based on a two-tailed $t$ test a

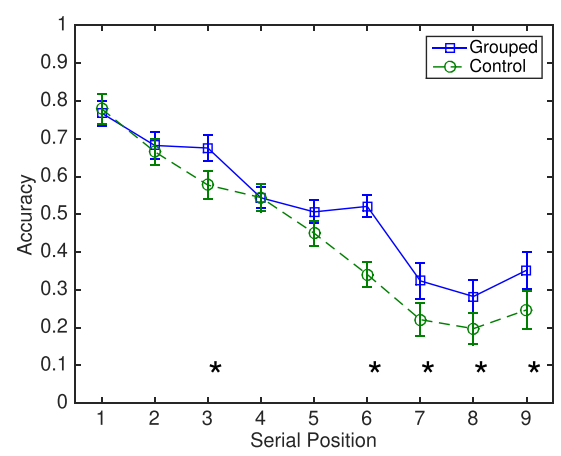

b

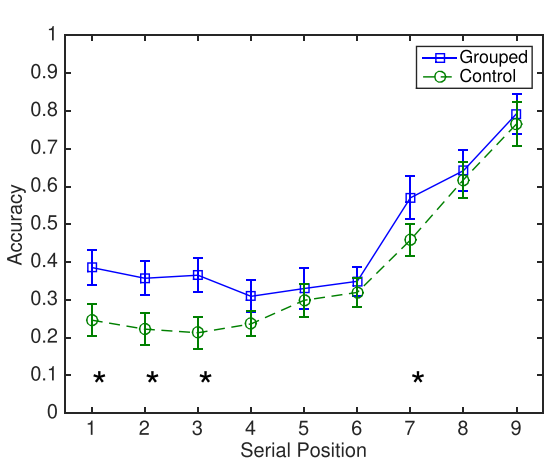

c

Grouped-Control

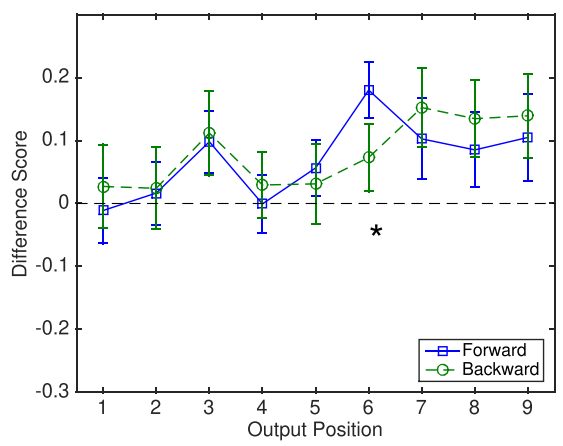


Table 1 The best-fitting LME model for recall accuracy (proportion correct) with Serial Position as a factor (a) or with Output Position as a factor (b) for Experiment 1

$\begin{array}{lll}\text { Effect } & \text { Estimate (SE) } & p\end{array}$

a Analysis using Serial Position $(\mathrm{BIC}=127169)$

Main effects

\begin{tabular}{|c|c|c|c|}
\hline & Intercept & $1.02(0.10)^{*}$ & $<.001$ \\
\hline & Grouping & $-0.054(0.13)$ & 0.678 \\
\hline & Direction & $-2.47(0.06)^{*}$ & $<.001$ \\
\hline & Serial Position & $-0.27(0.01)^{*}$ & $<.001$ \\
\hline \multicolumn{4}{|l|}{ Interactions } \\
\hline & Grouping $\times$ Direction & $0.78(0.08)^{*}$ & $<.001$ \\
\hline & Grouping $\times$ Serial Position & $0.08(0.01)^{*}$ & $<.001$ \\
\hline & Direction $\times$ Serial Position & $0.40(0.01)^{*}$ & $<.001$ \\
\hline & Grouping $\times$ Direction $\times$ Serial Position & $-0.15(0.01)^{*}$ & $<.001$ \\
\hline \multicolumn{4}{|c|}{ b Analysis using Output Position $(\mathrm{BIC}=127150)$} \\
\hline \multicolumn{4}{|l|}{ Main effects } \\
\hline & Intercept & $0.99(0.10)^{*}$ & $<.001$ \\
\hline & Grouping & $-0.005(0.13)$ & 0.971 \\
\hline & Direction & $-1.03(0.04)^{*}$ & $<.001$ \\
\hline & Output Position & $-0.26(0.01)^{*}$ & $<.001$ \\
\hline \multicolumn{4}{|l|}{ Interactions } \\
\hline & Grouping $\times$ Output Position & $0.08(0.01)^{*}$ & $<.001$ \\
\hline & Direction $\times$ Output Position & $0.12(0.01)^{*}$ & $<.001$ \\
\hline & \multicolumn{3}{|c|}{$\Delta \mathrm{BIC}=19$, favoring the model with Output Position } \\
\hline
\end{tabular}

Estimate - the corresponding regression coefficient, along with its SE (standard error of the mean). BIC favors the model with Output Position; note that (a) includes a three-way interaction (highlighted in boldface) that is absent from (b). ${ }^{*} p<0.05$

Fig. 3 Inter-response time as a function of output position for forward recall (a) and backward recall (b) for Experiment 1. Inter-response time difference of Grouped-Control as a function of output position for forward and backward recall (c). Error bars denote $95 \%$ confidence intervals based on standard error of the mean. Significant differences between Grouped and Control condition are denoted by $* p<0.05$, based on a two-tailed $t$ test a

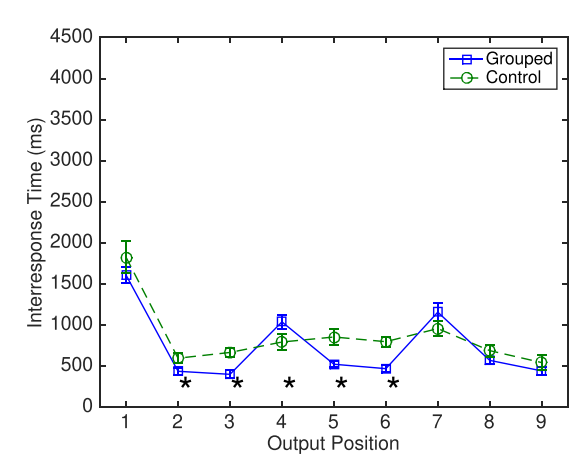

b

Backward Recall

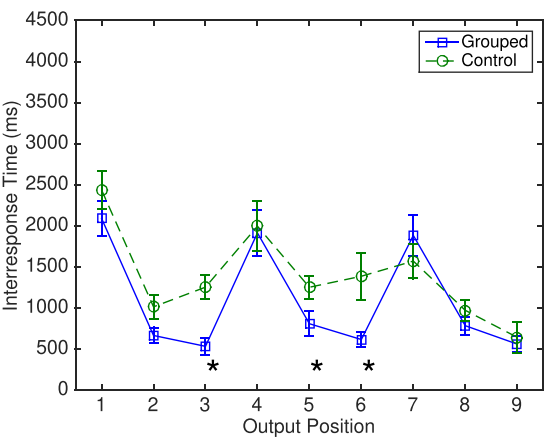

c

Grouped-Control

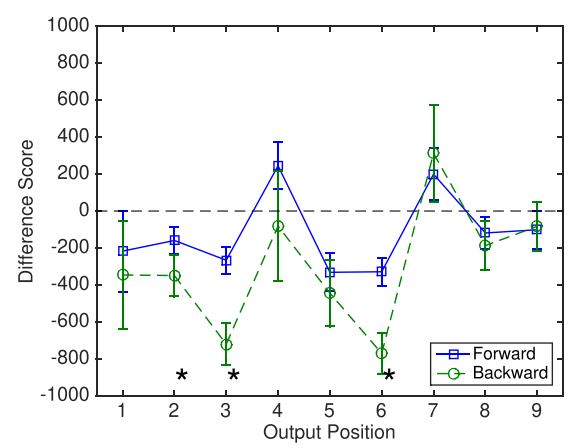


times were overall longer in the Backward than Forward direction (Table 2). The scalloping that is considered evidence of subjective chunking based on temporally grouped presentation was evident in both Forward and Backward recall. In fact, it was even more pronounced in the Backward direction, and even clearly evident in the Backward Control participants (Table 2).

\section{Within-list intrusions}

A favorite finding that has been used to support the standard, two-dimensional positional-coding account of temporal grouping is the increase in interpositions (an item recalled within the wrong group, but nonetheless, at the correct within-group position) and relative decrease in adjacent transpositions (an item exchanged with its neighbor) from control to grouped lists (Henson, 1998; Lee \& Estes, 1981; Wickelgren, 1964). We also replicated the classic effects of grouping on within-list intrusion types, for forward serial recall (Fig. 4 and Table 4). However, for backward recall, grouping did not produce an increase in the rate of interpositions (Fig. 4), supported by a significant Error Type $\times$ Direction $\times$ Grouping interaction in the best-fitting LME model (Table 3 ).

Taking the standard argument further, if the advantage conferred by grouping is due to cueing with a twodimensional position code, and the presence of interposition errors signifies the usage of within-group position as a cue (Wickelgren, 1964), then the more a participant commits interpositions (expressed as a proportion of all withinlist intrusions), the greater their serial-recall accuracy should be. A positive correlation between interposition rate and accuracy is predicted for grouped conditions. The correlation was indeed positive and significant, but only for the Forward Control condition $r(38)=0.33, p=$ 0.039. For the Backward Control condition, the correlation was significant but negative, $r(34)=-0.37, p=$ 0.025 , opposite the prediction. Moreover, for the Grouped conditions, the correlations were both quite small and nonsignificant, (Forward: $r(39)=-0.063, p=0.70$; Backward: $r(40)=-0.127, p=0.42)$; thus, we found no simple relationship between interposition rates and the advantage due to grouping (Table 4).

\section{Experiment 2}

\section{Methods}

\section{Participants}

A total of 110 undergraduate students from introductory psychology courses at the University of Alberta participated in exchange for partial course credit. Sample size was determined to be comparable to that of Experiment 1, but we anticipated greater sensitivity since one factor was within, rather than between subjects, thus requiring a smaller sample. Participants gave informed consent, had normal or corrected-to-normal vision and learned English before age 6. Participants were run in groups of about 10 to 15 persons, with random assignment to testing groups.

\section{Materials \& procedures}

The procedure was identical to Experiment 1, except that participants were told sometimes they need to recall in forward order, and sometimes they need to recall in backward order, and they need to follow the instruction on the computer screen. The text reminder in Experiment 1 was replaced with the directionality cue ("Type the list in FORWARD order" or "Type the list in BACKWARD order"), after each list had been presented for study.

\section{Results}

Participants recalling fewer than two items on average (making their data uninformative with respect to serialorder, scalloping effects, etc.) were excluded from the analysis: Grouped: 3; Control: 14. The number of included participants was significantly different between conditions, $\chi^{2}(1)=40.33 p<.001$.

Table 2 The best-fitting LME model for log inter-response time with Output Position as a factor for Experiment 1

\begin{tabular}{lllr}
\hline Main effects & & $\chi^{2}$ & \\
& Intercept & & \\
& Grouping & $16.8^{*}$ & $<.001$ \\
& Direction & $29.9^{*}$ & $2890^{*}$ \\
Interactions & Output Position & & $<.001$ \\
& & $39.93^{*}$ & $<.001$ \\
\hline
\end{tabular}

The $\chi^{2}$ column reports $\chi^{2}$ from Wald test. $* p<0.05$ 
Fig. 4 Proportion of adjacent errors and interposition errors for each condition. The error bars denote the $95 \%$ confidence interval based on standard error of the mean a

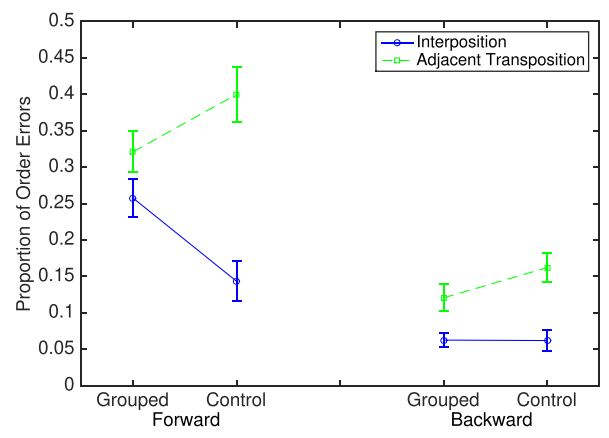

b

Experiment 2

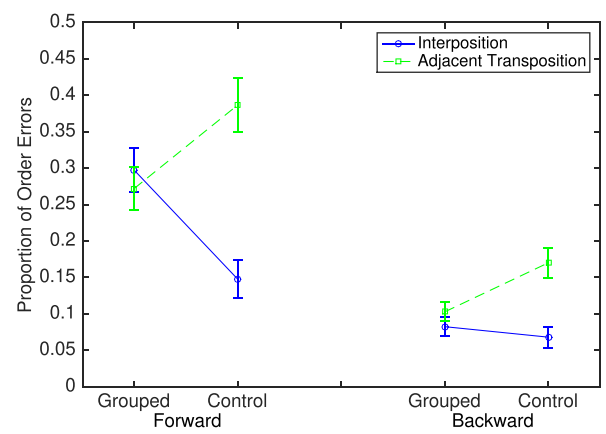

As in Experiment 1, we first look at Grouping and Direction collapsing across Input/Output Positions for both accuracy and inter-response times of correct recalls (Fig. 1). Direction is a within-subjects factor and Grouping is a between-subjects factor. The basic pattern of results was similar to Experiment 1, with main effects of Grouping and Direction.

Figure 5 plots serial-position curves for all four conditions. The Grouped participants showed the characteristic scalloping pattern. Once again, the overall serial-position curves for backward recall are nearly (although not precisely) mirror images of the corresponding curves for forward recall and the effects of grouping are approximate mirror images as well. This visual impression that the benefit of grouping is a function of output position is further confirmed by the plot of Grouped-Control accuracy as a function of output position (Fig. 5c), which is quite similar for backward as for forward recall.

As in Experiment 1, we fitted linear mixed effects models with either Serial Position or Output Position (Table 5), along with Grouping and Direction. The best-fitting linear mixed effects models using Serial Position as a factor found a Grouping $\times$ Direction $\times$ Serial Position three-way interaction. Using Output Position instead, the best-fitting model did not include Grouping $\times$ Direction $\times$ Output Position. As in Experiment 1, the BIC values show that the best-fitting model using Output Position (absent a three-way interaction) fit better than the best-fitting model with Serial Position (which includes a three-way interaction): $\triangle \mathrm{BIC}=$ 21.

Inter-response time as a function of output position looks similar to Experiment 1 (Fig. 6). All groups show the characteristic slow initiation time. After the first recall, the Grouped participants had longer recall latencies across group boundaries than Control participants, in exchange for shorter latencies within groups for both forward and backward recall directions. As in Experiment 1, interresponse times were longer overall in the backward than forward direction. The scalloping that is considered evidence of subjective chunking was evident in both forward and backward directions, and it was, again, more pronounced in the backward direction, and in the backward Control condition (Table 6). It may be worth noting that the inter-response times were slower than in Experiment 1 (Fig. 1), perhaps due to a form of task-switching cost.

Table 3 The best-fitting LME model for error proportion scores (proportion of errors normalized by within-list errors) with Grouping, Direction, and Error Type as factors, for Experiment 1

\begin{tabular}{|c|c|c|c|}
\hline \multirow[t]{5}{*}{ Main effects } & & $\chi^{2}$ & $\mathrm{p}$ \\
\hline & Intercept & & \\
\hline & Grouping & 0.25 & 0.615 \\
\hline & Direction & $383.22 *$ & $<.001$ \\
\hline & Error Type & $70.37^{*}$ & $<.001$ \\
\hline \multicolumn{4}{|l|}{ Interactions } \\
\hline & Error Type $\times$ Direction & $3.94 *$ & $¡ .047$ \\
\hline & Error Type $\times$ Grouping & $94.85^{*}$ & $<.001$ \\
\hline & Direction $\times$ Grouping & $6.12 *$ & 0.013 \\
\hline & Direction $\times$ Error Type $\times$ Grouping & $26.45^{*}$ & $<.001$ \\
\hline
\end{tabular}

The $\chi^{2}$ column reports $\chi^{2}$ from Wald test. *p $<0.05$ 
Table 4 Rates of interposition and adjacent errors (relative to all within-list intrusions) for each condition

\begin{tabular}{|c|c|c|c|c|}
\hline & \multicolumn{2}{|l|}{ Forward } & \multicolumn{2}{|l|}{ Backward } \\
\hline & Grouped & Control & Grouped & Control \\
\hline \multicolumn{5}{|l|}{ Experiment 1} \\
\hline Adjacent Transpositions & $32.11 \%$ & $39.99 \%$ & $12.06 \%$ & $16.21 \%$ \\
\hline Interpositions & $25.75 \%$ & $14.32 \%$ & $6.25 \%$ & $6.19 \%$ \\
\hline \multicolumn{5}{|l|}{ Experiment 2} \\
\hline Adjacent Transpositions & $27.21 \%$ & $38.68 \%$ & $10.28 \%$ & $17.01 \%$ \\
\hline Interpositions & $29.71 \%$ & $14.74 \%$ & $8.23 \%$ & $6.79 \%$ \\
\hline
\end{tabular}

Cell values are calculated by dividing the number of adjacent transposition and interposition errors by total number of errors per condition

\section{Recall-direction mismatches}

We were concerned that manipulating recall direction within subjects might have confused participants, making it difficult for them to perfectly comply, and sometimes recalling a list in the wrong direction. To check the potential impact of such recall direction mismatches, we implemented a conservative recall directionality measure: the sum of differences between the serial positions of all adjacent recalls for a list. A positive value was considered evidence of an attempt to recall forward, and a negative value, an attempt to recall backward. Across all lists, $18.08 \%$ of backward recall trials were suspected possible wrong directions (recalled forward) and $8.08 \%$ of forward recall lists were suspected possible wrong directions (recalled backward). When we replotted the serial-position and output-position curves with those (conservatively) suspected wrong-direction lists removed, this did not change the major features of the plots (Fig. S1 and S2 in Supplementary Materials).

\section{Within-list intrusions}

We conducted within-list intrusion analysis following the same methods in Experiment 1, and replicated those findings. For forward serial recall (Fig. $4 \mathrm{~b}$ and
Fig. 5 Recall accuracy as a function of serial position for forward recall (a) and backward recall (b) for Experiment 2. Accuracy difference of Grouped-Control as a function of output position for forward and backward recall (c). Error bars denote $95 \%$ confidence intervals based on standard error of the mean. $* p<0.05$, based on a two-tailed $t$ test a

Forward Recall

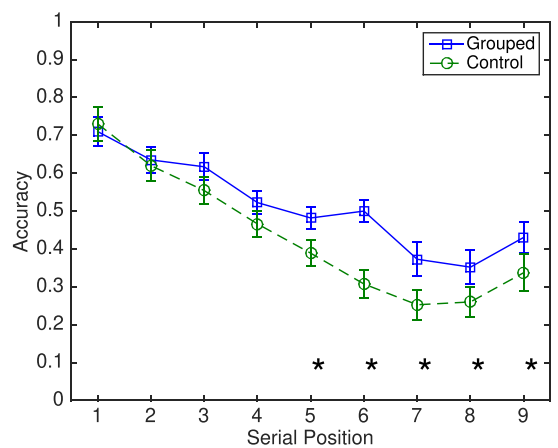

b

Backward Recall

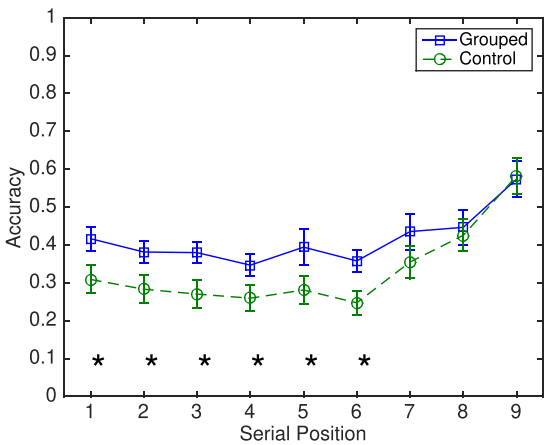

c

Grouped-Control

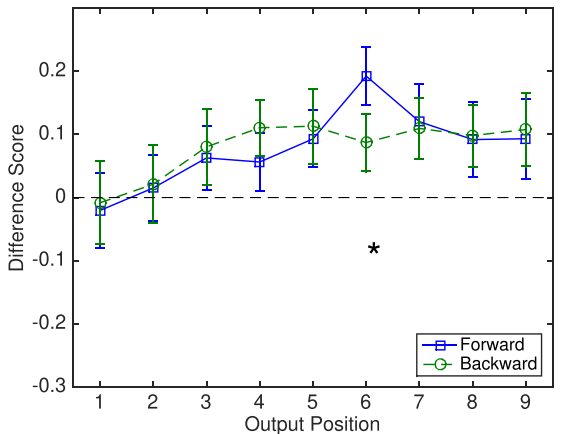


Table 5 The best-fitting LME model for recall accuracy (proportion correct) with Serial Position as a factor (a) or with Output Position as a factor (b) for Experiment 2

Effect $\quad$ Estimate (SE)

a Analysis using Serial Position $(\mathrm{BIC}=75195)$

Main effects

\begin{tabular}{|c|c|c|c|}
\hline & Intercept & $0.78(0.10)^{*}$ & $<.001$ \\
\hline & Grouping & $0.20(0.14)$ & 0.163 \\
\hline & Direction & $-2.70(0.057)^{*}$ & $<.001$ \\
\hline & Serial Position & $-0.28(0.0070)^{*}$ & $<.001$ \\
\hline \multicolumn{4}{|l|}{ Interactions } \\
\hline & Grouping $\times$ Direction & $0.89(0.076)^{*}$ & $<.001$ \\
\hline & Grouping $\times$ Serial Position & $0.086(0.0095)^{*}$ & $<.001$ \\
\hline & Direction $\times$ Serial Position & $0.45(0.010)^{*}$ & $<.001$ \\
\hline & Grouping $\times$ Direction $\times$ Serial Position & $-0.18(0.013)^{*}$ & $<.001$ \\
\hline \multicolumn{4}{|c|}{ b Analysis using Output Position $(\mathrm{BIC}=75174)$} \\
\hline \multicolumn{4}{|c|}{ Main effects } \\
\hline & Intercept & $0.79(0.10)^{*}$ & $<.001$ \\
\hline & Grouping & $0.19(0.14)$ & 0.164 \\
\hline & Direction & $-1.07(0.036)^{*}$ & $<.001$ \\
\hline & Output Position & $-0.28(0.0060)^{*}$ & $<.001$ \\
\hline \multicolumn{4}{|l|}{ Interactions } \\
\hline & Grouping $\times$ Output Position & $0.088(0.0067)^{*}$ & $<.001$ \\
\hline & Direction $\times$ Output Position & $0.12(0.0066)^{*}$ & $<.001$ \\
\hline & \multicolumn{3}{|c|}{$\Delta \mathrm{BIC}=21$, favoring the model with Output Position } \\
\hline
\end{tabular}

Estimate - the corresponding regression coefficient, along with its SE (standard error of the mean). BIC favors the model with Output Position; note that (a) includes a three-way interaction (highlighted in boldface) that is absent from (b). $* p<0.05$

Fig. 6 Inter-response time as a function of output position for forward recall (a) and backward recall (b) for Experiment 2.

Inter-response time difference of Grouped-Control as a function of output position for forward and backward recall (c). Error bars denote $95 \%$ confidence intervals based on standard error of the mean. Significant differences between Grouped and Control condition are denoted by $* p<0.05$, based on a two-tailed $t$ test a

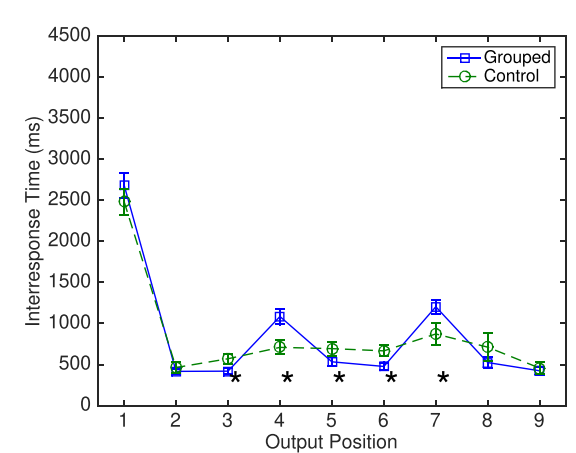

b

Backward Recall

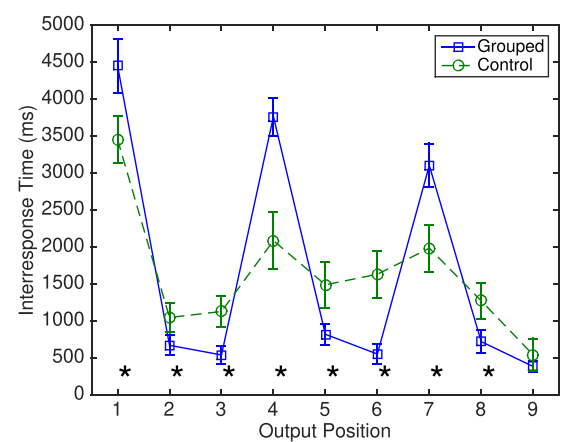

c

Grouped-Control

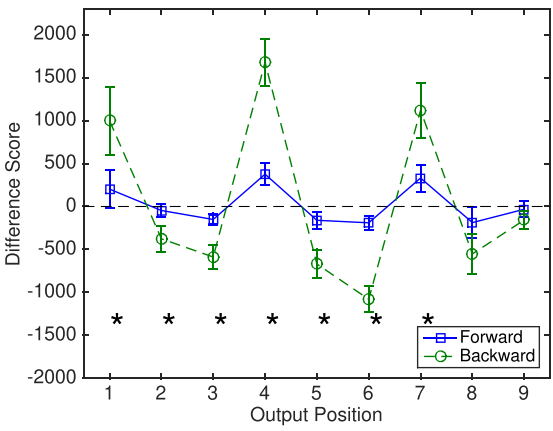


Table 6 The best-fitting LME model for log inter-response time with Output Position as a factor for Experiment 2

\begin{tabular}{|c|c|c|c|}
\hline \multirow[t]{5}{*}{ Main effects } & & $\chi^{2}$ & $\mathrm{p}$ \\
\hline & Intercept & & \\
\hline & Grouping & 0.55 & 0.458 \\
\hline & Direction & $895.31^{*}$ & $<.001$ \\
\hline & Output Position & $3641.12^{*}$ & $<.001$ \\
\hline \multicolumn{4}{|l|}{ Interactions } \\
\hline & Group $\times$ Direction & 8.51 & 0.004 \\
\hline & Direction $\times$ Output Position & $18.46^{*}$ & $<.001$ \\
\hline & Grouping $\times$ Output Position & $0.27^{*}$ & 0.604 \\
\hline & Grouping $\times$ Direction $\times$ Output Position & $19.86^{*}$ & $<.001$ \\
\hline
\end{tabular}

The $\chi^{2}$ column reports $\chi^{2}$ from Wald test. $* p<0.05$

Table 4), grouping reduced adjacent transposition errors and increased interposition errors, but for backward recall, grouping did not produce an increase in the rate of interpositions Fig. 4b), supported by a significant Error Type $\times$ Direction $\times$ Grouping interaction in the best-fitting LME model (Table 7). In line with Experiment 1, the correlation between proportion of interpositions and recall accuracy was either non-significant or even (nominally) negative for the Grouped conditions (Forward: $r(50)=-0.076, p=$ 0.59; Backward: $r(50)=-0.58, p<0.001)$. The correlation was positive, although small and non-significant for the Forward Control condition $(r(39)=0.20, p=0.20)$, was significant but negatively correlated for the Backward Control condition $(r(39)=-0.50, p<0.001)$. As with Experiment 1, these results are hard to reconcile with the hypothesis that committing interpositions is related to the mechanism by which temporal grouping enhances serial recall accuracy.

\section{Discussion of experiments}

In sum, inter-response time data suggest participants did appear to subjectively chunk temporally grouped lists into three groups of three items, in the backward as well as forward direction. Grouping improved accuracy for backward as well as forward recall. However, apparently inconsistent with the standard positional-coding account, the benefit to accuracy was not a function of serial position, but rather, of output position. Interpositions were observed, but they were not more prevalent in the temporally grouped condition for backward recall. Interpositions were not reliably associated with better accuracy in Grouped conditions, so their relationship to the effects of temporal grouping on accuracy and inter-response times is not clear. Finally, these key characteristics were found both when recall-direction was known to participants prior to studying a list (Experiment 1) and when recall-

Table 7 The best-fitting LME model for error proportion scores (proportion of errors normalized by within-list errors) with Grouping, Direction, and Error Type as factors, for Experiment 2

\begin{tabular}{|c|c|c|c|}
\hline \multirow[t]{5}{*}{ Main effects } & & $\chi^{2}$ & \\
\hline & Intercept & & \\
\hline & Grouping & 0.0014 & 0.970 \\
\hline & Direction & $382.65^{*}$ & $<.001$ \\
\hline & Error Type & $89.06^{*}$ & $<.001$ \\
\hline \multicolumn{4}{|l|}{ Interactions } \\
\hline & Error Type $\times$ Direction & $6.90 *$ & 0.009 \\
\hline & Error Type $\times$ Grouping & $90.97 *$ & $<.001$ \\
\hline & Direction $\times$ Grouping & $12.64 *$ & $<.001$ \\
\hline & Direction $\times$ Error Type $\times$ Grouping & $25.31 *$ & $<.001$ \\
\hline
\end{tabular}

The $\chi^{2}$ column reports $\chi^{2}$ from Wald test. $* p<0.05$ 
direction was not instructed until after each list had been studied (Experiment 2). This challenges the idea that in Experiment 1, participants in the backward recall-direction were simply reverse-coding list-position or within-group position. It also provides a clue that the mechanism by which temporal grouping enhances serial-recall accuracy may be located at the recall, rather than the study phase.

\section{Models}

The results of both experiments suggested the advantage due to temporal grouping depends on output position, not serial position. At first blush, the empirical pattern seems at odds with current positional-coding accounts of temporal grouping effects. However, positional-coding models do have ways in which phenomena could respond to output position. For example, in SIMPLE, as recall proceeds, study-test intervals for all items lengthen. Because SIMPLE acts on the logarithm of the study-test interval, ${ }^{3}$ itemdiscriminability worsens at later output positions. Second, response suppression (the tendency of participants to avoid repeating items during recall; Duncan \& Lewandowsky, 2005) enables accuracy of one recall to influence probability of later recalls. We first checked whether the current formulation of SIMPLE already possesses the ability to produce output-order effects of temporal grouping, by fitting SIMPLE to the data from Experiment 1. There may be good intuitive reasons to expect this model would be insufficient. However, we felt it is important to give this model a fair chance to fit the data for several reasons. First, the principle of parsimony entails that one should not jump to a more complicated account when a simpler account is sufficient (for known data). Second, even in this basic version of SIMPLE, recall probability is determined by temporal discriminability. Thus, the temporal pattern of presentation might be sufficient to produce the kind of advantage due to temporal grouping we observed. Third, in addition to temporally patterned presentation, participants' own responses were temporally patterned. We found it intriguing to consider the possibility that temporally patterned responses might have some separate cause (e.g., participants feeling compelled to mimic the rhythm of presentation), but could then have a spillover effect, causing

\footnotetext{
${ }^{3}$ As discussed by Brown et al. (2007), findings of absent isolation effects in forward serial recall (e.g., Lewandowsky and Brown, 2005; Lewandowsky et al., 2006) lead to the idea that participants virtually ignore the temporal dimension during serial recall, in the same paragraph (p. 562), they nonetheless explain that they retain their focus on the temporal dimension. Indeed, several of the serialrecall phenomena they fit seem to rely upon (temporal) recency and relative time differences, not just ordinal position. We adopted their assumptions, having adapted their MATLAB model code, and acknowledge that Brown and colleagues' formulation of SIMPLE, as well as our own, could be fairly challenged or modified on this basis.
}

temporal discriminability to evolve over the course of recall, in a characteristic way that could, itself, be the cause of the effects of temporal grouping on probability of recall.

Next, we added within-group position cueing to the model to test whether Brown et al.'s (2007) twodimensional cueing account could be sufficient to explain both the forward and backward serial recall data. Finally, we incorporated response suppression into both the twodimensional and one-dimensional models, to test whether response suppression could be critical to understanding the precise pattern of effects of temporal grouping on recall probability.

\section{Modelling methods}

The model was adapted from the MATLAB code used to simulate temporal grouping effects by Brown et al. (2007). SIMPLE starts with the assumption that memory is driven by discriminability of presentation times of items (Brown et al., 2007), relative to time of test. Thus, psychological time, $M_{i}=\log \left(\right.$ test - study time). Similarity, $\eta_{i, j}$, between the psychological times of two items, $i$ and $j$, is:

$\eta_{i, j}=e^{-c\left|M_{i}-M_{j}\right|}$,

where $c$ parameterizes the level of confusibility of presentation times and $|\cdot|$ denotes absolute value (magnitude). To accommodate temporal grouping in the second model variant, we follow Brown et al. (2007) and assume a second dimension of discriminability, due to position within-group. Each item thus has one within-group position value; as a function of serial position, these values, $P_{i}$, are 3, 2, 1, 3, 2, 1, 3, 2, 1 (inverted just as time is relative to time of test). Again, following Brown et al. (2007), we assume that confusibility is a function of the L1 (taxicab metric) distance between the two-dimensional (time, within-group position) values:

$\eta_{i, j}=e^{-c\left(w_{t}\left|M_{i}-M_{j}\right|+w_{p}\left|P_{i}-P_{j}\right|\right)}$,

where $w_{t}$ and $w_{p}$ are weighting parameters to vary the relative reliance on time versus within-group position, respectively, and $w_{t}+w_{p}=1$. Because spontaneous grouping is often observed even when lists are presented ungrouped (e.g., Martin and Noreen, 1974; Madigan, 1980; Jou, 2011), $w_{p}$ is allowed to vary between grouped and control lists, with the expectation that $w_{p}$ will not necessarily be zero for Control participants, but will at least be greater for Grouped than Control conditions.

During recall, the model probes successively in an attempt to retrieve the first item, then the second, etc. Thus, for forward recall, the model probes with the time (recency) of serial position 1, then with the updated recency of serial position 2, proceeding through serial position $L$. Following Bireta et al. (2010), backward recall starts by probing with 
the recency of serial position $L$, then with the updated serial position $L-1$, down through serial position 1 . The probability of recalling item $j$ in position $i$ is based on the confusibility between $i$ and $j$. First, the discriminability of item $j$, given position $i$ as the cue, is:

$$
D(j \mid i)=\frac{\eta_{i, j}}{\sum_{k=1}^{L} \eta_{i, k}},
$$

These $D$ values are passed through a sigmoid function to produce recall probabilities:

$$
P(j \mid i)=\frac{1}{1+\exp [-s(D(j \mid i)-\theta)]},
$$

where $s$ and $\theta$ determine the slope and threshold of the sigmoid, respectively. In case the sum of probabilities for a given recall position exceed 1, which is logically impossible, the probabilities are renormalized by that sum to fix the total recall probability over all list items to 1 . We thus tacitly assume that if probabilities do not sum to 1, the remaining instances are omissions (but might also be thought of as extra-experimental intrusions).

SIMPLE was developed to explain accuracy data, but not yet to address latency data. However, because the model includes a temporal dimension, timing of both list presentation and responses can critically influence model output. Brown et al. (2007) did not have inter-response time values for the grouping data they fit (Hitch, Burgess, Towse, and Culpin, 1996); in lieu of such data, they assumed that response times increased exponentially, $n^{1.5} \mathrm{~s}$ for response $n$. Because of the primary importance of interresponse times in determining temporal discriminability in SIMPLE, we fit SIMPLE using the mean inter-response times observed in our data set. These inter-response times were not exactly those plotted in Figs. 3 and 6, because those plot inter-response times for correct recalls only; for model fitting, we used the total time from study to time of recall, regardless of accuracy of the prior responses. List length and presentation times were the same in the model as in the experiment.

Response suppression Finally, although SIMPLE, like other positional-coding models, assumes independentcueing, there is one more way in which recall can change systematically over the course of the recall sequence. Because participants rarely repeat an item during serial recall (e.g., Duncan \& Lewandowsky, 2005; Henson, 1998; Vousden \& Brown, 1998), many models have incorporated a mechanism for response suppression, making it impossible or unlikely to recall an item that has already been produced as a response (e.g., Brown et al., 2000; Burgess \& Hitch, 1999; Henson, 1998; Lewandowsky \& Murdock, 1989).
Thus, as recall progresses, the size of the response set will diminish. This suggests that if accuracy is increased early in recall, those correct recalls will, helpfully, reduce the response set available for subsequent recalls, making it easier to produce accurate responses later in the sequence. Thus, an early benefit will not only persist, but could accumulate over the course of recalls. We added response suppression to the model as follows. ${ }^{4}$ 1) For each recall, compute the probability of each list item not having yet been recalled. That is the product of $1-p$ up to the current recall operation. 2) In the denominator of the discriminability equation: for each list item within the summation, multiply by the probability that this item was not yet recalled. 3 ) Calculate probabilities as usual (via the sigmoid function). 4) Multiply the final probabilities by the probability that each item has not yet been recalled. Note that this also avoids the need to check for probabilities exceeding 1 .

\section{Model fitting}

SIMPLE was fit to each participant's data individually, with the SIMPLEX algorithm (Nelder \& Mead, 1965), using an adaptation of MATLAB's fminsearch function and root-mean-squared-deviation (RMSD) as the measure of fitness. The ranges searched were designed to encompass and extend the ranges of parameters reported by Brown et al. (2007), Table 1: $c=[0.45,30], \theta=[0,1], s=[1,100]$ and the within-group position weights were searched over the allowable range [0, 0.99]. For a given model, we fit the model to each participant 100 times and report the best-fitting model across those 100 runs, summarized across participants. Because BIC, log-likelihood and RMSD are all monotonically related to one another, when the number of free parameters is held constant, optimizing for RMSD produces the same result as optimizing for loglikelihood or BIC. BIC values were, in turn, computed via an estimation of log-likelihood, following Burnham and Anderson (2004). Bear in mind that our purpose here is not to find the "correct" model of immediate serial recall and temporal grouping effects. Rather, the model fits will indicate whether our findings do or do not pose a challenge to a model that incorporates that standard positional-cueing account of temporal grouping. The parameter searches give the model the opportunity to provide its best quantitative fit, but the primary way we evaluate the model is by checking whether it produces the key qualitative effects found in the data; most notably: an increase in the benefit of temporal

\footnotetext{
${ }^{4}$ To our knowledge, published versions of SIMPLE have never incorporated response suppression, although Brown et al. (2007) commented that they had done so.
} 
grouping over the course of the recall sequence for both forward and backward recall directions.

\section{Modeling results}

SIMPLE with a single dimension At its heart, SIMPLE explains accuracy in serial recall as primarily dependent upon discriminability of study-test intervals. We noted that the pattern of study-test intervals differs for grouped lists, and moreover, study times (inter-response times) are temporally patterned. This led us to propose that the absolutely simplest plausible explanation would be that the effects of temporal grouping on accuracy are a direct consequence of the temporal patterning of study and recall times. We thus first fit a 1-D version of SIMPLE, where the only dimension was (study-test) time.

Plotted in Fig. 7, although the quantitative fit is better for backward than forward recall, it is a bit worse for Grouped than Control data. More concerning, the model underpredicts the amount of scalloping of the Grouped data, particularly in the forward direction, and underestimates primacy while overestimating recency accuracy in the forward direction. Finally, the model underpredicts the difference between Grouped and Control accuracy late in recall.

\section{SIMPLE with a second-dimension coding within-group order} Because the 1-D model failed to produce scalloping of accuracy, we next added within-group position as a cue, following Brown et al. (2007). Plotted in Fig. 8, it is clear that this model fit quantitatively better for the grouped conditions (and interestingly, nearly no change in BIC values for the Control conditions). This model produced more scalloping, particularly in the Grouped conditions. The model also succeeded fairly well in capturing the increase in advantage for grouping with increasing output a

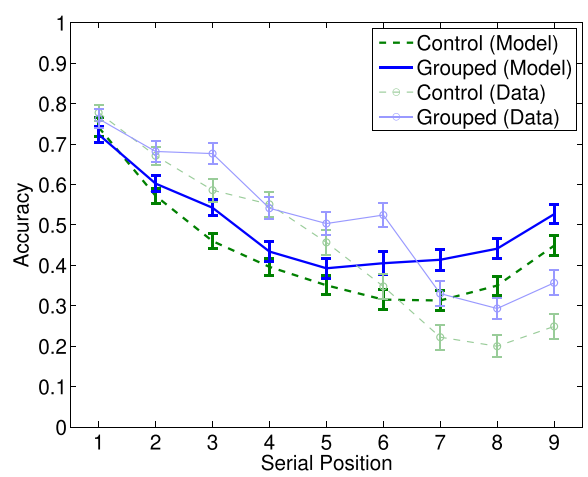

c

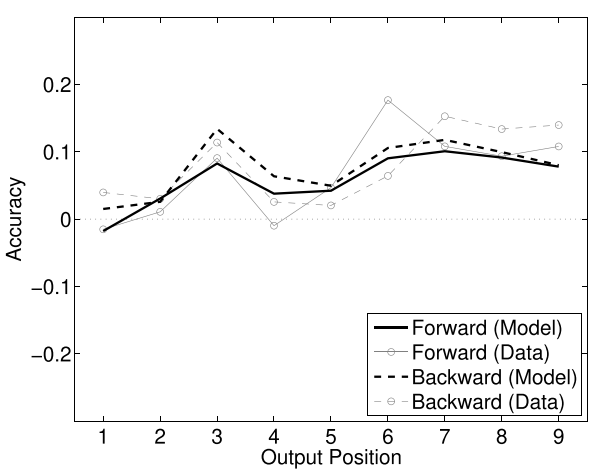

b

d

\begin{tabular}{|rcc|}
\multicolumn{4}{c}{$(M \pm S D)$} \\
\hline & Control & Grouped \\
Forward & $-31.15 \pm 11.58$ & $-30.84 \pm 10.21$ \\
Backward & $-42.97 \pm 10.33$ & $-39.53 \pm 10.60$ \\
\hline
\end{tabular}

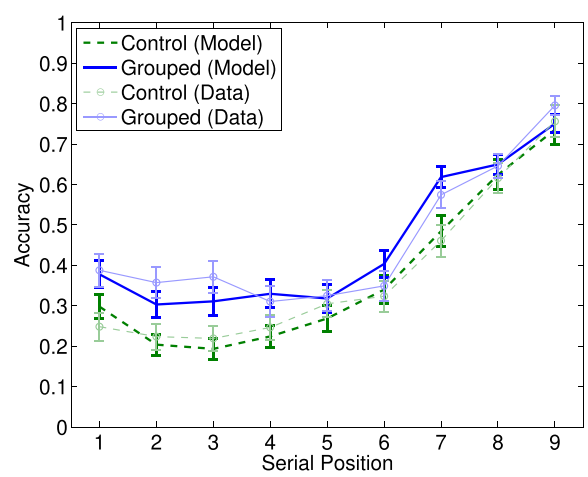

BIC Values

$(M \pm S D)$

\section{$-1$}

\begin{tabular}{|rcc|}
\multicolumn{3}{c}{$\Delta \mathrm{BIC}$} \\
\hline & Control & Grouped \\
Forward & 15.80 & 16.67 \\
Backward & 0.81 & 4.97 \\
\hline
\end{tabular}

across participants. (d) BIC values (upper table) and mean $\triangle$ BIC compared to the best model (lower table). In panels a-c, the data from Experiment 1 are plotted in lighter shades 
a

Forward Recall

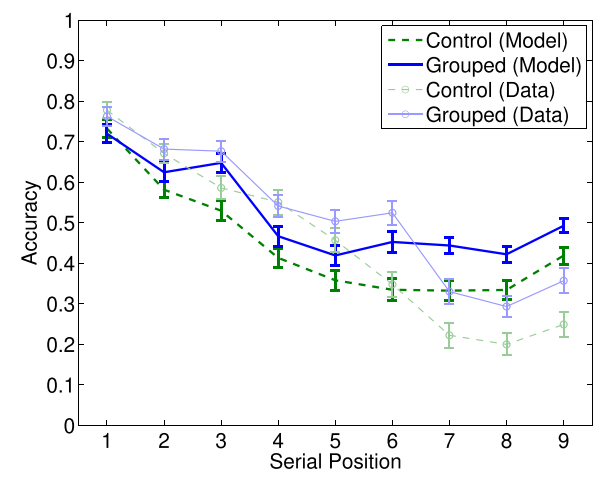

c

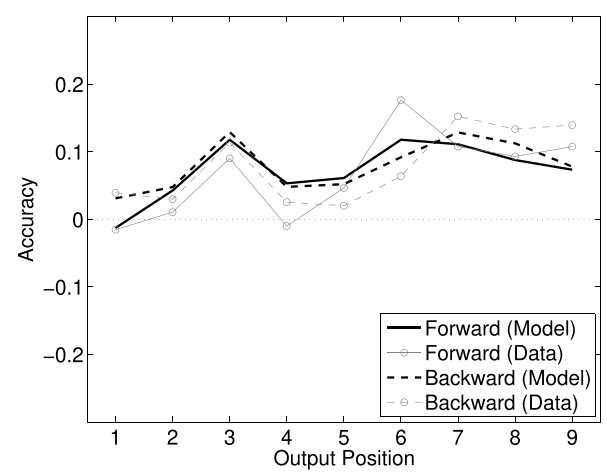

Fig. 8 Best-fitting parameter sets for the Two-dimensional model, fit to individual participants. Recall accuracy as a function of serial position for forward recall (a) and backward recall (b) and for the Grouped-Control difference (c). Error bars plot standard error of the

position. Where the model fell short was, again, in underestimating early-list accuracy and overestimating latelist accuracy for the forward recall direction. In addition, the model produced nearly-null effects of grouping at the initial two response positions, but not quite as equivalent accuracy levels as in the data.

Response suppression Next, we added response suppression to the 2-D model (see Modelling Methods). Note that our implementation adds no free parameters to the model. That is because we assume response suppression is complete, and does not relax over the course of the recall period, as shown by Duncan and Lewandowsky (2005).

As is evident in Fig. 9, response suppression, combined with 2-D cueing, produced better BIC values than the previous two models in all conditions, and with a mean $\Delta \mathrm{BIC}>2$ in all conditions except Backward Control. Panels a-c show that the model behavior approached the data quite well, capturing all the noteworthy qualitative features of the data, including near-null effects of grouping at output positions 1, 2, 4 and 5, and an overall trend of increasing benefit due to grouping with increasing output b

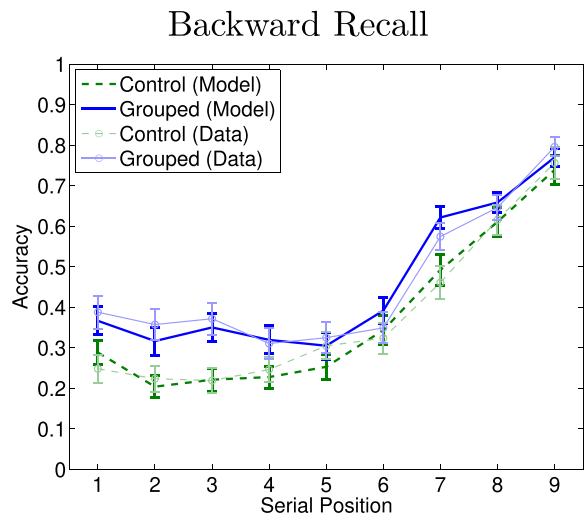

d

BIC Values

$(M \pm S D)$

\begin{tabular}{|ccc|}
\hline & Control & Grouped \\
Forward & $-30.64 \pm 11.51$ & $-34.41 \pm 14.49$ \\
Backward & $-42.52 \pm 11.76$ & $-42.01 \pm 20.77$ \\
\hline
\end{tabular}

\begin{tabular}{|rcc|}
\multicolumn{3}{c}{$\Delta$ BIC } \\
\hline & Control & Grouped \\
Forward & 16.31 & 13.10 \\
Backward & 1.26 & 2.49 \\
\hline
\end{tabular}

mean across participants. (d) BIC values (upper table) and mean $\triangle \mathrm{BIC}$ compared to the best model (lower table). In panels $\mathbf{a}-\mathbf{c}$, the data from Experiment 1 are plotted in lighter shades

position. In sum, this model produced the lowest BIC values and the best qualitative fits.

To follow up on the excellent fit of this model, we next examine the distributions of best-fitting parameter values, which are plotted in Fig. 10. First, $c$, which controls distinctiveness along the temporal dimension, fit to higher values for Grouped than Control for Forward recall participants, but the reverse for the Backward recall participants. On the whole, the model estimated more temporal distinctiveness for Backward than Forward-recall participants. However, this parameter also traded off with the sigmoid parameters, so there may be some dependencies amongst these three parameters. Interestingly, the model fit higher values of the within-chunk cueing parameter, $w_{p}$, for Grouped than Control participants, and this was the case for both the Forward and Backward recall data. Moreover, in each direction, there was nearly stochastic dominance; at nearly every percentile the value of $w_{p}$ was greater for the grouped participant than the corresponding control participant. This is consistent with the idea that withinchunk cueing explains the difference between Grouped and Control data. 
a

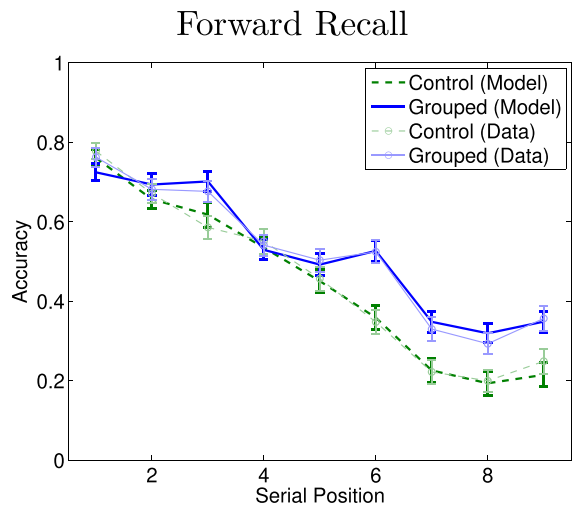

c

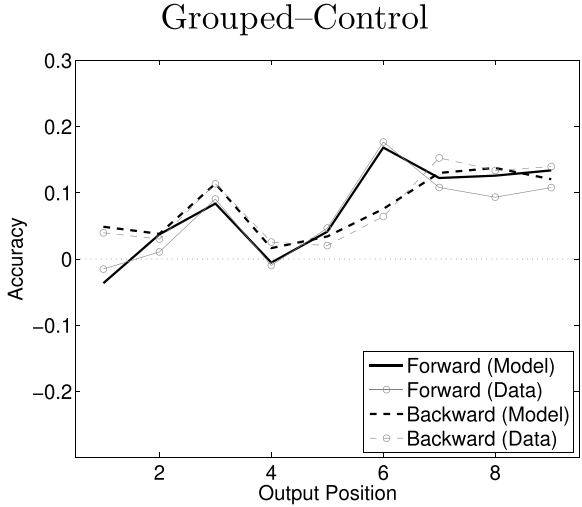

b

d
Backward Recall

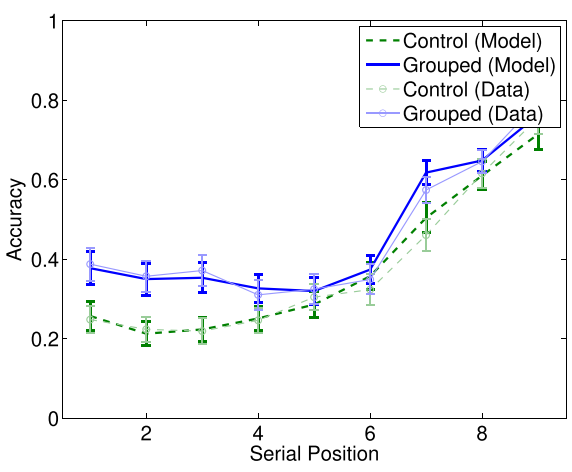

BIC Values

$(M \pm S D)$

\begin{tabular}{|ccc|}
\hline & Control & Grouped \\
Forward & $-46.95 \pm 7.78$ & $-47.51 \pm 7.81$ \\
Backward & $-43.78 \pm 10.62$ & $-44.50 \pm 16.81$ \\
\hline
\end{tabular}

\begin{tabular}{|rcc|}
\multicolumn{3}{c}{$\Delta$ BIC } \\
\hline & Control & Grouped \\
Forward & 0.00 & 0.00 \\
Backward & 0.00 & 0.00 \\
\hline
\end{tabular}

bars plot standard error of the mean across participants. (d) BIC values (upper table) and mean $\triangle \mathrm{BIC}$ compared to the best model (lower table). In panels $\mathbf{a}-\mathbf{c}$, the data from Experiment 1 are plotted in lighter shades

the relative weighting of within-chunk to temporal cueing and the tendency to make interposition errors.

Response Suppression and the 1-D model Finally, we wanted to check whether response suppression, alone, might be largely responsible for the strong fit to the data-namely, with the within-group cueing mechanism removed. When we added response suppression to the one-dimensional model (Fig. 11), BIC values for the Control conditions were only slightly worse, but BIC values were far worse for the Grouped conditions. Qualitatively, the shapes of the output-position effects were fairly similar to the data, deviating notably by too much of an advantage for Grouped participants at output position 3 (serial position 7) and too little at output position 7 (serial position 3). For the Forward conditions, the fit was quite good for Control participants, but completely missed the scalloping effect for Forward, Grouped participants, even with an inverted $\mathrm{U}$ shape for the first group (positions 1-3). Thus, even with the help of response suppression to produce effects 
Temporal distinctiveness $(c)$

a

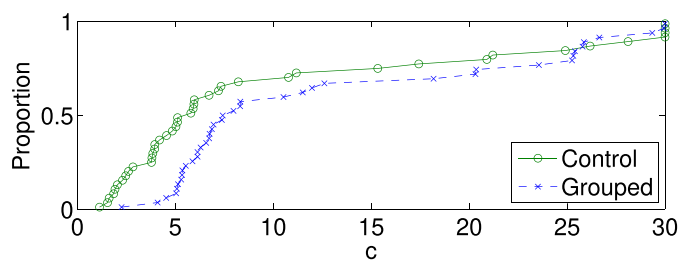

b

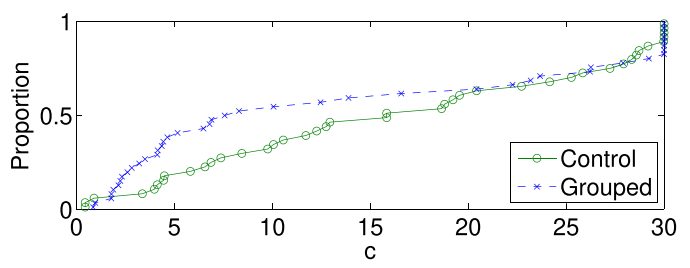

Sigmoid slope $(s)$

c

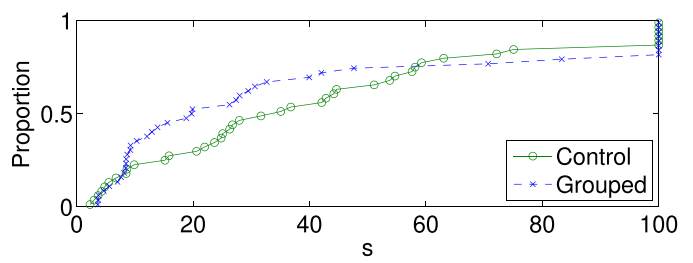

d

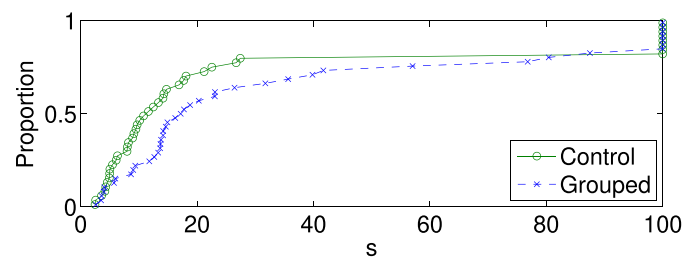

Sigmoid threshold $(\theta)$

e

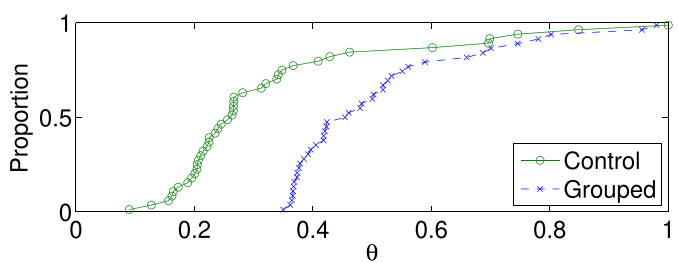

f

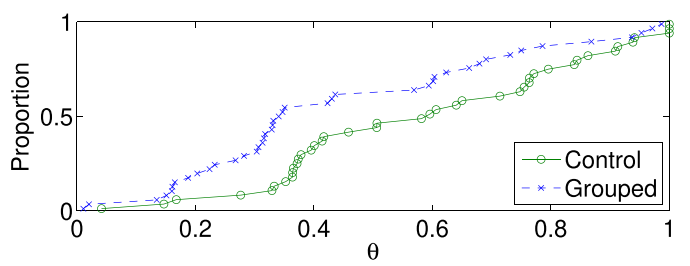

Within-chunk cueing weight $\left(w_{p}\right)$

g

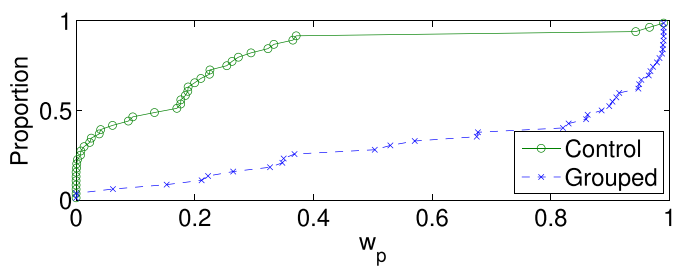

$\mathbf{h}$

Backward

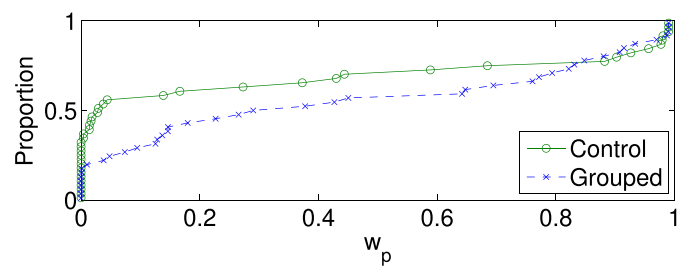

Fig. 10 Cumulative distribution functions of best-fitting parameters for the 2-D model with response suppression

dominated by output order, relying on temporal patterning during presentation and during recall could not adequately fit the Grouped data.

\section{General discussion}

Our novel condition, backward serial recall of temporally grouped lists, produced the characteristic scalloping effect, in both accuracy and inter-response time measures, extending this type of finding to backward recall. Importantly, the backward recall condition revealed that the benefit of grouping was driven by output order rather than input order. Foreknowledge (in Experiment 1, compared to Experiment 2) did not change this characteristic of the result (resembling the effect of word length reported by Surprenant et al. (2011) but differing from the effect of spatial manual tapping reporting by Guitard et al. (2019)). This provided a clue that the most likely locus of the advantage of temporal grouping is during recall rather than during study.

The overall dominance of output position on recall accuracy and near-mirror symmetry of forward and backward serial recall seemed, at first, to challenge positional-coding models, and in particular, the twodimensional positional cueing account of temporal grouping effects. Indeed, our first models, based on SIMPLE, were severely inadequate accounts of the data, particularly of the Grouped conditions. When we added response suppression, the model with both temporal and within-chunk-position cueing fit the data quite well, both quantitatively and 
a

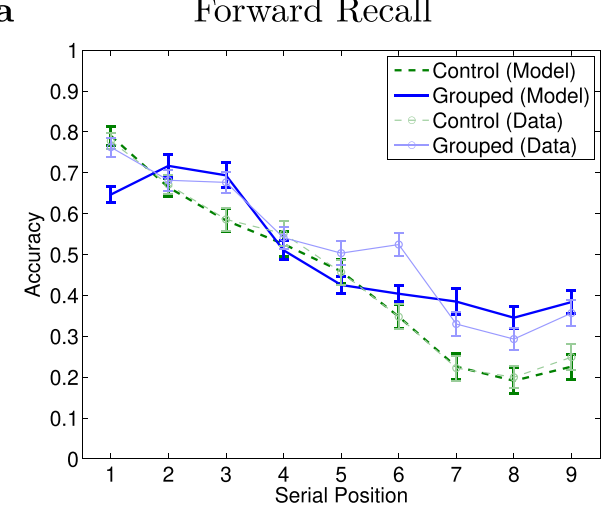

c

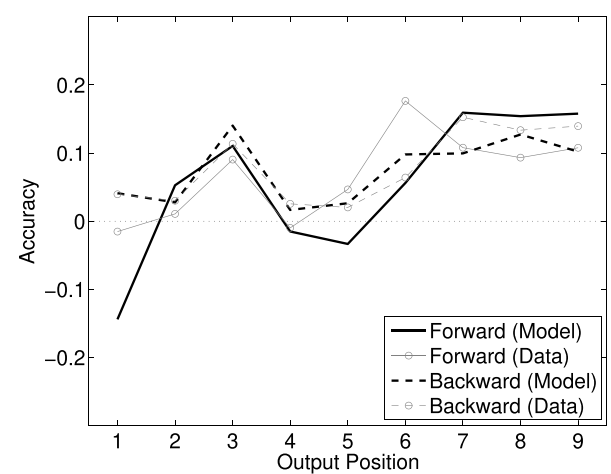

b

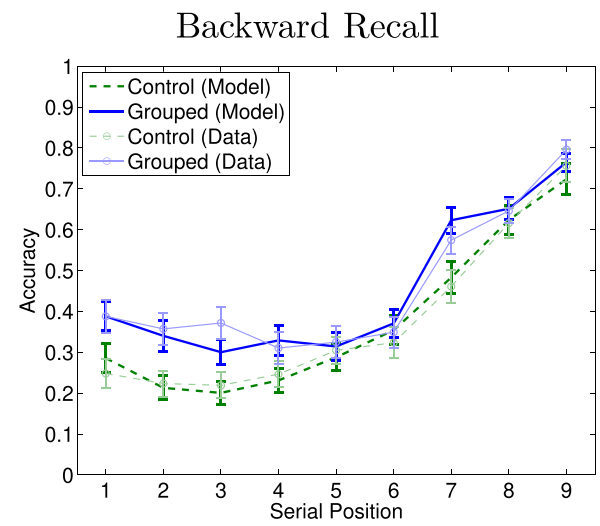

d
BIC Values

$(M \pm S D)$

\section{Control}

Grouped

Forward $\quad-45.84 \pm 6.09 \quad-36.78 \pm 8.50$

Backward $\quad-43.10 \pm 9.47 \quad-39.70 \pm 9.70$

\begin{tabular}{|rcc|}
\multicolumn{3}{c}{$\Delta \mathrm{BIC}$} \\
\hline & Control & Grouped \\
Forward & 1.11 & 10.73 \\
Backward & 0.68 & 4.80 \\
\hline
\end{tabular}

Fig. 11 Best-fitting parameter sets for the one-dimensional with response suppression model, fit to individual participants. Recall accuracy as a function of serial position for forward recall (a) and backward recall (b) and for the grouped-control difference (c). Error bars plot standard error of the mean across participants. d BIC values (upper table) and mean $\triangle \mathrm{BIC}$ compared to the best model (lower table). In panels $\mathbf{a}-\mathbf{c}$, the data from Experiment 1 are plotted in lighter shades

Brown et al., 2000; Burgess and Hitch, 1999; Henson, 1998), and to determine whether response suppression is a critical ingredient for those models as well. Associative chaining models were argued to be ruled out for immediate serial recall of relatively short lists (e.g., Henson, 1998), but many of the early arguments against associative chaining have been challenged (e.g., Caplan, 2015; Caplan et al., 2015; Farrell, 2006; Hulme et al., 2003; Kahana et al., 2010; Serra \& Nairne, 2000; Solway et al., 2012), suggesting that a re-consideration of the evidence against chaining models may be in order. Such a chaining model would need to be updated, to include symmetric associations and remote associations, for example. An associative chaining account of our findings might be plausible, although this would entail a large amount of work, as no update to associative chaining models has been tested against a comprehensive set of benchmark findings since Lewandowsky and Murdock (1989). One well-known challenge to an associative chaining model would be to produce within-list intrusion phenomena that suggest positional cueing; namely, interpositions and protrusions, which are intrusions from prior lists that are recalled in 
the same position as their original presentation position, more often than expected by chance (Fischer-Baum \& McCloskey, 2015; Henson, 1998; 1999; Osth \& Dennis, 2015). While these phenomena should not be overlooked, it is not yet clear how they should be accommodated in any model. First, protrusions (and prior-list intrusions) are rare, as these studies have shown. Second, the greater rate of interpositions for grouped versus control lists replicated for forward but not for backward serial recall, even in Experiment 2, suggesting that even a positional cue might fail to capture the interaction of interposition rate with recall direction without modification. Finally, as has been reasoned, hybrid models should be considered (Caplan, 2015), incorporating both associative and positional or ordinal cueing. It could be the case that the benefit of temporal grouping to serial recall accuracy derives from one mechanism (e.g., associative cueing) while protrusions and the elevated interposition rate in the forward, but not backward, direction, are due to the contribution of positional/ordinal cueing. Hierarchical models, wherein chunks are stored distinctly from isolated list items, might be able to accommodate our findings. One such model, by Farrell (2012), may require some modification; currently, it includes the assumption that chunks are retrieved in forward order. This would seem contradicted by the striking symmetry of our effects of temporal grouping on forward and backward recall directions, but this remains to be tested directly.

There is an abundance of research that has suggested participants can re-code lists into smaller units, often called "chunks," where a chunk is assumed to be retrieved in an all-or-none step (e.g., Anderson and Matessa, 1997; Anderson et al., 1998; Farrell, 2012; Johnson, 1969; 1972; Lee \& Estes, 1981; Martin \& Noreen, 1974; Miller, 1956; Murdock, 1995; 1997). Our data do not speak directly to this possibility. We note that the experimental paradigms that have led to this conception of chunking are quite different, and are typically transfer designs, either based on pre-experimental knowledge or two-list transfer paradigms (e.g., Chekaf et al., 2016; Johnson, 1969; Thalmann et al., 2019) rather than temporal grouping. The possibility that temporal grouping effects might be related to those transfer effects is intriguing, but would also be best investigated directly in future studies.

In recent years, there has been an accelerating interest in comparing backward serial recall with forward serial recall. Results seem split as to whether a given experimental manipulation is consistent between recall directions (e.g., Bireta et al., 2010; Guérard et al., 2012; Guérard \& Saint-Aubin, 2012; Guitard et al., 2019; Manning \& Pacifici, 1983) or dissociates recall directions (e.g., Baker et al., 2012; Olivia et al., 2018; Guitard et al., 2019; Li \& Lewandowsky, 1993; 1995; Madigan, 1971;
Norris et al., 2019; Richardson, 2007; Ritchie et al., 2015; Surprenant et al., 2011; Thomas et al., 2003). Our temporal-grouping manipulation would add to the former, suggesting a common mechanism, across recall direction, for the effect of temporal grouping on recall. However, this does not change the fact that backward serial recall differs from forward serial recall in other ways. Evidence from self-reported strategy usage, validated with accuracy and response-time serial-position effects, suggests that participants may use a mix of strategies to perform backward, as well as forward recall (Norris, Hall, \& Gathercole, 2019). Although our model fits found a single model to be sufficient to fit individual participants as a group, some fit better than others; thus, although overall, temporal grouping effects were well explained by a combination of explicit within-group position-cueing along with repetition suppression, it is possible that this could be modulated by particular strategies. A complete model would need to accommodate both commonalities and dissociations between recall directions.

In sum, both experiments revealed the effects of temporal grouping on immediate serial recall to be primarily functions of output position and not serial position. This presents a novel constraint on mathematical models of serial recall. However, with the addition of the well-supported process of response suppression, a temporal-distinctiveness model, incorporating within-chunk position cueing, was able to fit the empirical pattern quite closely. In conclusion, the characteristic scalloping pattern observed in immediate serial recall of temporally grouped items appears to be driven by a combination of within-group cueing and the accumulation of benefits to accuracy due to response suppression.

Acknowledgements Portions of this work were reported in a dissertation presented by Yang S. Liu to the Department of Psychology, University of Alberta, 2015. Supported in part by the Natural Sciences and Research Council of Canada. The authors thank Madhawa Alahakoon for assistance in conducting the second experiment.

Open Practices Statement The data and materials for all experiments are available at https://osf.io/evmct/?view only=be14d124209442f8882e476f812965b6, and none of the experiments was preregistered.

\section{References}

Anderson, J. R., Bothell, D., Lebiere, C., \& Matessa, M. (1998). An integrated theory of list memory. Journal of Memory and Language, 38, 341-380.

Anderson, J. R., \& Matessa, M. (1997). A production system theory of serial memory. Psychological Review, 104(4), 728-748.

Baayen, R. H. (2007). LanguageR (R package on CRAN version 1.1). http://cran.r-project.org/web/packages/languageR/index.html. 
Baayen, R. H., Davidson, D. J., \& Bates, D. M. (2008). Mixed-effects modeling with crossed random effects for subjects and items. Journal of Memory and Language, 59, 390-412.

Baayen, R. H., \& Milin, P. (2010). Analyzing reaction times. International Journal of Psychological Research, 3(2), 12-28.

Baker, R., Tehan, G., \& Tehan, H. (2012). Word length and age influences on forward and backward immediate serial recall. Memory \& Cognition, 40(1), 40-51.

Bates, D. M. (2005). Fitting linear mixed models in R. $R$ News, 5 , 27-30.

Bates, D. M., \& Sarkar, D. (2007). lme4: Linear mixed-effects models using s4 classes (version 0.999375-39) [Computer software and manual]. http://cran.r-project.org/web/packages/lme4/.

Bireta, T. J., Fry, S. E., Jalbert, A., Neath, I., \& Surprenant, A. M. (2010). Backward recall and benchmark effects of working memory. Memory \& Cognition, 38(3), 279-291.

Brown, G. D. A., Neath, I., \& Chater, N. (2007). A temporal ratio model of memory. Psychological Review, 114(3), 539-576.

Brown, G. D. A., Preece, T., \& Hulme, C. (2000). Oscillator-based memory for serial order. Psychological Review, 107(1), 127-181.

Burgess, N., \& Hitch, G. J. (1999). Memory for serial order: A network model of the phonological loop and its timing. Psychological Review, 106(3), 551-581.

Burnham, K. P., \& Anderson, D. R. (2004). Multimodel inference: Understanding AIC and BIC in model selection. Sociological Methods \& Research, 33(2), 261-304.

Caplan, J. B. (2015). Order-memory and association-memory. Canadian Journal of Experimental Psychology, 69(3), 221-232.

Caplan, J. B., Madan, C. R., \& Bedwell, D. J. (2015). Itemproperties may influence item-item associations in serial recall. Psychonomic Bulletin \& Review, 22(2), 483-489.

Chan, M., Ross, B., Earle, G., \& Caplan, J. B. (2009). Precise instructions determine participants' memory search strategy in judgments of relative order in short lists. Psychonomic Bulletin \& Review, 16(5), 945-951.

Chekaf, M., Cowan, N., \& Mathy, F. (2016). Chunk formation in immediate memory and how it relates to data compression. Cognition, 155, 96-107.

Duncan, M., \& Lewandowsky, S. (2005). The time course of response suppression: No evidence for a gradual release from inhibition. Memory, 13(3/4), 236-246.

Ebbinghaus, H. (1885/1913). Memory: A contribution to experimental psychology. New York: Teachers College, Columbia University.

Farrand, P., \& Jones, D. (1996). Direction of report in spatial and verbal serial short-term memory. Quarterly Journal of Experimental Psychology, 49A(1), 140-158.

Farrell, S. (2006). Mixed-list phonological similarity effects in delayed serial recall. Journal of Memory and Language, 55, 587-600.

Farrell, S. (2012). Temporal clustering and sequencing in short-term memory and episodic memory. Psychological Review, 119(2), 223271.

Fischer-Baum, S., \& McCloskey, M. (2015). Representation of item position in immediate serial recall: Evidence from intrusion errors. Journal of Experimental Psychology: Learning, Memory, and Cognition.

Fox, J., \& Weisberg, S. (2011). An R companion to applied regression, (2nd ed.). Sage: Thousand Oaks CA. Retrieved from http:// socserv.socsci.mcmaster.ca/jfox/Books/Companion.

Geller, A. S., Schleifer, I. K., Sederberg, P. B., Jacobs, J., \& Kahana, M. J. (2007). PyEPL: A crossplatform experiment-programming library. Behavior Research Methods, 39(4), 950-958.

Guérard, K., \& Saint-Aubin, J. (2012). Assessing the effect of lexical variables in backward recall. Journal of Experimental Psychology: Learning, Memory, and Cognition, 38(2), 312-324.

Guérard, K., Saint-Aubin, J., Burns, S. C., \& Chamberland, C. (2012). Revisiting backward recall and benchmark memory effects: A reply to Bireta et al. (2010). Memory \& Cognition, 40, 388-407.
Guitard, D., Saint-Aubin, J., Poirier, M., Miller, L. M., \& Tolan, A. (2019). Forward and backward recall: Different visuospatial processes when you know what's coming. Memory \& Cognition, 48(5), 111-126.

Haberlandt, K., Lawrence, H., Krohn, T., Bower, K., \& Thomas, J. G. (2005). Pauses and durations exhibit a serial position effect. Psychonomic Bulletin \& Review, 12(1), 152-158.

Henson, R. N. A. (1998). Short-term memory for serial order: The Start-End Model. Cognitive Psychology, 36(2), 73-137.

Henson, R. N. A. (1999). Positional information in short-term memory: Relative or absolute? Memory \& Cognition, 27(5), 915927.

Hitch, G. J., Burgess, N., Towse, J. N., \& Culpin, V. (1996). Temporal grouping effects in immediate recall: A working memory analysis. Quarterly Journal of Experimental Psychology, 49A(1), 116-139.

Hulme, C., Stuart, G., Brown, G. D. A., \& Morin, C. (2003). High- and low-frequency words are recalled equally well in alternating lists: Evidence for associative effects in serial recall. Journal of Memory and Language, 49(4), 500-518.

Johnson, N. F. (1969). Chunking: Associative chaining versus coding. Journal of Verbal Learning and Verbal Behavior, 8(6), 725-731.

Johnson, N. F. (1972). Organization and the concept of a memory code. In Melton, A. W., \& Martin, E. (Eds.) Coding processes in human memory, (pp. 125-159). Washington: Winston \& Sons.

Jou, J. (2011). Two paradigms of measuring serial-order memory: Two different patterns of serial position functions. Psychological Research Psychologische Forschung, 75(3), 202-213.

Kahana, M. J., Mollison, M. V., \& Addis, K. M. (2010). Positional cues in serial learning: The spin-list technique. Memory \& Cognition, 38(1), 92-101.

Lashley, K. S. (1951). The problem of serial order in behavior. In Jeffress, L. A. (Ed.) Cerebral mechanisms in behavior, (pp. 112146). New York: Wiley.

Lee, C. L., \& Estes, W. K. (1981). Item and order information in shortterm memory: Evidence for multilevel perturbation processes. Journal of Experimental Psychology: Learning, Memory, and Cognition, 7(3), 149-169.

Lewandowsky, S., \& Brown, G. D. A. (2005). Serial recall and presentation schedule: A micro-analysis of local distinctiveness. Memory, 13(3/4), 283-292.

Lewandowsky, S., Brown, G. D. A., Wright, T., \& Nimmo, L. M. (2006). Timeless memory: Evidence against temporal distinctiveness models of short-term memory for serial order. Journal of Memory and Language, 54, 20-38.

Lewandowsky, S., \& Murdock, B. B. (1989). Memory for serial order. Psychological Review, 96(1), 25-57.

Li, S.-C., \& Lewandowsky, S. (1993). Intralist distractors and recall direction: Constraints on models of memory for serial order. Journal of Experimental Psychology: Learning, Memory, and Cognition, 19(4), 895-908.

Li, S.-C., \& Lewandowsky, S. (1995). Forward and backward recall: Different retrieval processes. Journal of Experimental Psychology: Learning, Memory, and Cognition, 21(4), 837-847.

Liu, Y. S. (2015). Human order memory: Insights from the relative-order task (Unpublished doctoral dissertation). University of Alberta.

Liu, Y. S., Chan, M., \& Caplan, J. B. (2014). Generality of a congruity effect in judgements of relative order. Memory \& Cognition, 42(7), 1086-1105.

Madigan, S. (1980). The serial position curve in immediate serial recall. Bulletin of the Psychonomic Society, 15(5), 335-338.

Madigan, S. A. (1971). Modality and recall order interactions in short-term memory for serial order. Journal of Experimental Psychology, 87(2), 294-296.

Manning, S. K., \& Pacifici, C. (1983). The effects of a suffixprefix on forward and backward serial recall. American Journal of Psychology, 96(1), 127-134. 
Martin, E., \& Noreen, D. L. (1974). Serial learning: Identification of subjective subsequences. Cognitive Psychology, 6, 421-435.

Miller, G. A. (1956). The magical number seven, plus or minus two: Some limits on our capacity for processing information. Psychological Review, 63(2), 81-97.

Murdock, B. B. (1995). Developing TODAM: Three models for serial-order information. Memory \& Cognition, 23(5), 631-645.

Murdock, B. B. (1997). Context and mediators in a theory of distributed associative memory (TODAM2). Psychological Review, 104(4), 839-862.

Nelder, J. A., \& Mead, R. (1965). A simplex method for function minimization. Computer Journal, 7, 308-313.

Ng, H. L. H., \& Maybery, M. T. (2002). Grouping in short-term verbal memory: Is position coded temporally? Quarterly Journal of Experimental Psychology, 55A(2), 391-424.

Norris, D., Hall, J., \& Gathercole, S. E. (2019). How do we perform backward serial recall? Memory \& Cognition, 47(3), 519543.

Olivia, B., Saint-Aubin, J., Guérard, K., \& Pâquet, M. (2018). Are lexical factors immune to response modality in backward recall? The effects of imageability and word frequency. Canadian Journal of Experimental Psychology/Revue canadienne de psychologie expérimentale, 72(2), 105.

Osth, A. F., \& Dennis, S. (2015). Prior-list intrusions in serial recall are positional. Journal of Experimental Psychology: Learning, Memory, and Cognition, 41(6), 1893-1901.

Plant, R. R., \& Turner, G. (2009). Millisecond precision psychological research in a world of commodity computers: New hardware, new problems? Behaviour Research Methods, 41(3), 598-614.

Richardson, J. T. E. (2007). Measures of short-term memory: A historical review. Cortex, 43, 635-650.

Ritchie, G., Tolan, G. A., Tehan, G., Goh, H. E., Guérard, K., \& SaintAubin, J. (2015). Phonological effects in forward and backward serial recall: Qualitative and quantitative differences. Canadian Journal of Experimental Psychology, 69(1), 95-103.

Ryan, J. (1969a). Grouping and short-term memory: Different means and patterns of grouping. Quarterly Journal of Experimental Psychology, 21(2), 137-147.
Ryan, J. (1969b). Temporal grouping, rehearsal and short-term memory. Quarterly Journal of Experimental Psychology, 21(2), 148-155.

Serra, M., \& Nairne, J. S. (2000). Part-set cueing of order information: Implications for associative theories of serial order memory. Memory \& Cognition, 28(5), 847-855.

Solway, A., Murdock, B. B., \& Kahana, M. J. (2012). Positional and temporal clustering in serial order memory. Memory \& Cognition, 40(2), 177-190.

St. Clair Thompson, H. L., \& Allen, R. J. (2013). Are forward and backward recall the same? a dual-task study of digit recall. Memory \& Cognition, 41, 519-532.

Surprenant, A. M., Brown, M. A., Jalbert, A., Neath, I., Bireta, T. J., \& Tehan, G. (2011). Backward recall and the word length effect. American Journal of Psychology, 124(1), 75-86.

Thalmann, M., Souza, A. S., \& Oberauer, K. (2019). How does chunking help working memory? Journal of Experimental Psychology: Learning, Memory, and Cognition, 45(1), 37-55.

Thomas, J. G., Milner, H. R., \& Haberlandt, K. F. (2003). Forward and backward recall: Different response time patterns, same retrieval order. Psychological Science, 14(2), 169-174.

Tremblay, A. (2013). LMERConvenienceFunctions: A suite of functions to back-fit fixed effects and forward-fit random effects, as well as other miscellaneous functions (version 2.5) [Computer software and manual]. http://cran.r-project.org/web/ packages/LMERConvenienceFunctions/index.html.

Vousden, J. I., \& Brown, G. D. A. (1998). To repeat or not to repeat: The time course of response suppression in sequential behaviour. In Bullinaria, J. A., Glasspool, D. W., \& Houghton, G. (Eds.) Proceedings of the fourth neural computation and psychology workshop: Connectionist representations, (pp. 301-315). London: Springer.

Wickelgren, W. A. (1964). Size of rehearsal group and short-term memory. Journal of Experimental Psychology, 68(4), 413-419.

Wickelgren, W. A. (1967). Rehearsal grouping and hierarchical organization of serial position cues in short-term memory. Quarterly Journal of Experimental Psychology, 19(2), 97-102.

Publisher's note Springer Nature remains neutral with regard to jurisdictional claims in published maps and institutional affiliations. 